\title{
Reactive Balance Control in Immersive Visual Flows: 2D vs. 3D Virtual Stimuli
}

\author{
Olivier MARTIN ${ }^{\mathrm{a}, 1}$ and Jean-Dominique GASCUEL ${ }^{\mathrm{b}}$ \\ ${ }^{a}$ GIPSA-lab, Grenoble Universités, FRANCE \\ ${ }^{\mathrm{b}}$ ARTIS - LJK/INRIA Grenoble Rhône-Alpes, FRANCE
}

\begin{abstract}
The aim is to study the effects of $2 \mathrm{D}$ vs. $3 \mathrm{D}$ visual inputs on balance control. Ten subjects were fully immersed in virtual environment, using 10 different 2D/3D motion flow conditions. Analysis of visual and postural responses shows significant differences of reactivity in $3 \mathrm{D}$ versus $2 \mathrm{D}$.

Keywords. Balance control, virtual immersion, visual flow, performance measurement, adaptation
\end{abstract}

\section{Introduction}

The balance adaptation depends on efficient visual information processing in postural mechanisms. The visuo-postural control quickly integrates dynamic images properties to adapt motor mechanisms of balance. Our previous works have shown the ability to adapt movement and posture to visual changes [1] or visuomotor discordance [2] in virtual visual environments (VEs) with similar neuromotor processes as those of natural environment. However, the question about how the balance planning use visual cues is still unclear, mainly concerning the specific effects of 2D vs. 3D dynamics visual stimuli on the balance control. The goal of the study is to determine the effects of 2D vs. 3D visual inputs on ocular and balance responses. By analyzing the effects of the moving visual stimulation, this study also questions on the sensorimotor flexibility of the initial postural reaction and the online short-term balance adaptation in VEs.

\section{Methods}

Ten subjects aged from 22 to 42 participated in the study. Subjects stood within an immersive VE displayed on a 180 degrees curved screen (12x2 meters). Virtual visual stimulus consisted in randomly displayed star-like flow patterns. Ten conditions of stimuli were proposed : right, left, up and down (both in 2D and 3D), forward and backward (in 3D only). Each stimulus was presented during 20 seconds. Two randomized sessions of 10 trials per stimulus was performed, corresponding to 100 trials for each subject. A rest period occurred between sessions. Visual reactions (optokinetic nystagmus, $O K N$ ) and postural responses (kinematics and electromyography)

\footnotetext{
${ }^{1}$ Corresponding Author (olivier.martin@ujf-grenoble.fr)
} 
was recorded (200Hz). Data processing and analysis focused on the visual and balance adjustments parameters; A multi-factorial statistical design was applied to data.

\section{Results}

No subject mentioned immersive discomfort or after-effect. Results show that both visual and postural motor reactive responses to virtual scene motion occurs very quickly, specifically oriented to the Direction conditions of the visual flow in both $2 D$ and $3 D$ (fig. 1). The automatic OKN reactions was triggered from the first scene motions. For all Direction conditions, the frequency of the ocular responses decreased for 3D flow stimulus. At postural level, rapid postural adjustments allows to maintain the head stability level.

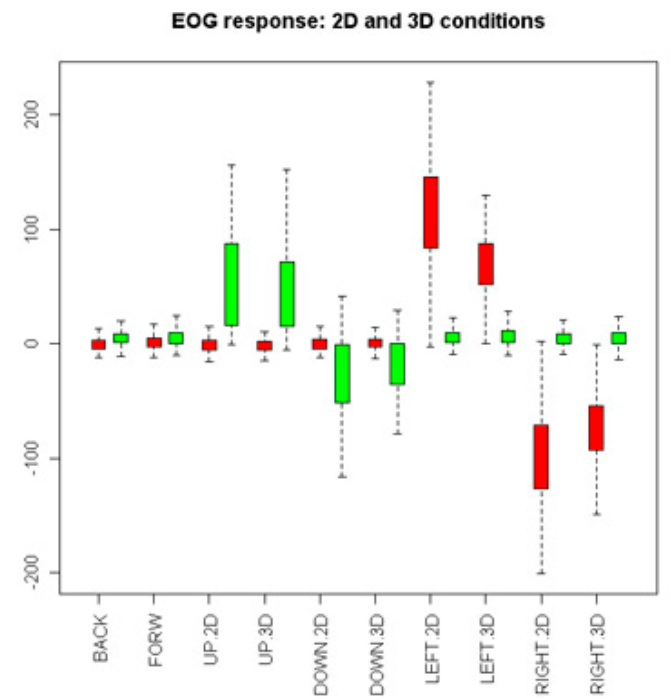

Figure 1. Ocular opto-kinetics responses signed OKN : count (left - right) and (up - down). Boxes represent central quartiles (50\%) for horizontal (green) and vertical (red) visual flows, for all $2 \mathrm{D}$ and $3 D$ conditions.

\section{Discussion and conclusion}

The study shows measurable reactivity to both vertical and horizontal, 2D and 3D flows in VEs. Furthermore, OKN count is higher for 2D than for 3D cues. We interpret this as the result of non-uniform motion of visual cues, slower for farthest ones, and even still at the vanishing point. It can also be hypothesized that cognitive load is added by the more complex perception of 3D flows. The combination of the automatic ocular responses with the rapid postural reactions is a global strategy for balance adaptation. The study proves that 3D visual flows (because they induce measurable and specific responses) are a pertinent tool for richer immersive protocols for balance analysis and rehabilitation.

\section{References}

[1] Martin O, Julian B, Boissieux L , Gascuel J-D, Prablanc C., Evaluating On-line Control of Goal Directed Arm Movement While Standing in Virtual Visual Environment, Journal of Visualization and Computer Animation, Vol.14 (2003), 253-260.

[2] Push A, Martin O, Coquillart S, HEMP - Hand-Displacement-Based Pseudo-Haptics : A study of a force field application and a behavioral analysis, International Journal of Human Computer Study, (in press 2009). 